\title{
The role of "spillover" in antibiotic resistance
}

Short title: Antibiotic resistance "spillover"

Scott W. Olesen ${ }^{1}$, Marc Lipsitch ${ }^{1,2}$, Yonatan H. Grad ${ }^{1,3}$

1 Department of Immunology and Infectious Diseases, Harvard T. H. Chan School of Public Health, Boston, Massachusetts 02115

2 Center for Communicable Disease Dynamics, Harvard T. H. Chan School of Public Health, Boston, Massachusetts 02115

3 Division of Infectious Diseases, Department of Medicine, Brigham and Women's Hospital, Harvard Medical School, Boston, Massachusetts 02115

Correspondence:

Yonatan Grad Harvard T. H. Chan School of Public Health 665 Huntington Ave, Building 1, Room 715, Boston, Massachusetts 02115 6174322275 ygrad@hsph.harvard.edu 


\section{ABSTRACT (240 words)}

2 Antibiotic use is a key driver of antibiotic resistance. Understanding the quantitative

3 association between antibiotic use and resulting resistance is important for predicting

4 future rates of antibiotic resistance and for designing antibiotic stewardship policy.

5 However, the use-resistance association is complicated by "spillover", in which one

6 population's level of antibiotic use affects another population's level of resistance via the

7 transmission of bacteria between those populations. Spillover is known to have effects

8 at the level of families and hospitals, but it is unclear if spillover is relevant at larger

9 scales. We used mathematical modeling and analysis of observational data to address

10 this question. First, we used dynamical models of antibiotic resistance to predict the

11 effects of spillover. Whereas populations completely isolated from one another do not

12 experience any spillover, we found that if even $1 \%$ of interactions are between

13 populations, then spillover may have large consequences: the effect of a change in

14 antibiotic use in one population on antibiotic resistance in that population could be

15 reduced by as much as $50 \%$. Then, we quantified spillover in observational antibiotic

16 use and resistance data from US states and European countries for 3 pathogen-

17 antibiotic combinations, finding that increased interactions between populations were

18 associated with smaller differences in antibiotic resistance between those populations.

19 Thus, spillover may have an important impact at the level of states and countries, which

20 has ramifications for predicting the future of antibiotic resistance, designing antibiotic

21 resistance stewardship policy, and interpreting stewardship interventions. 


\section{INTRODUCTION}

24 Antibiotic resistance is a major threat to public health (1). Outpatient antibiotic use,

25 which accounts for approximately $80 \%$ of human antibiotic use $(2,3)$, is considered a

26 principal driver of antibiotic resistance in the community (4). Understanding the

27 relationship between use and resistance is important because it allows accurate

28 predictions of the future of antibiotic resistance and goal-oriented antibiotic stewardship

29 policy. The use-resistance association has been previously characterized in many

30 ecological studies at the level of US states (5-7) and European countries $(8,9)$.

31 However, antibiotic resistance is a complex, temporally dynamic phenomenon (10-13),

32 and many factors complicate the use-resistance association, making what should be an

33 "obvious" connection sometimes difficult to identify and quantify (11). Even when

34 detected, observed use-resistance associations are sometimes weaker than might be

35 expected (14). One factor that could account for the difficulty in detecting use-resistance

36 associations in ecological studies and for the apparent weakness of such associations

37 is "spillover".

39 "Spillover" is a consequence of the fact that antibiotic-resistant and -susceptible bacteria

40 can be transmitted from person to person. Thus, one person's risk of an antibiotic

41 resistant infection depends on their own antibiotic use $(15,16)$ as well as the rates of

42 antibiotic use among their contacts (17). For example, one person's use of antibiotics

43 increases the risk of an antibiotic resistant infection among their family members (18-

44 21). As another example, hospitalized patients with no recent antibiotic use can have a

45 higher risk of resistance than people in the community with high antibiotic use (22) 
46 because antibiotic use and resistance in other hospitalized patients are high.

48 Spillover is important for three reasons. First, it means antibiotic resistance is not merely

49 a localized problem. It is well-understood that new resistance determinants can emerge

50 in one geography and spread globally $(23,24)$, but the role of spillover in determining the

51 levels of resistance in a given locale is not well-quantified. To what degree, for example,

52 can one US state expect that its antibiotic resistance levels are due to antibiotic use

53 within its borders, rather than in surrounding states? Second, spillover makes it difficult

54 to design antibiotic stewardship interventions and understand their results. For example,

55 if antibiotic use in one hospital changes, resistance might not change as expected

56 because of spillover, from the community or other hospitals into that hospital's patients.

57 Finally, spillover makes it difficult to interpret the results of controlled antibiotic

58 interventions, such as the effect of mass drug administration on antibiotic resistance

$59(25,26)$, when the intervention and control populations are not wholly epidemiologically

60 separate.

61

62 The effect of spillover should scale with the amount of interaction between populations.

63 If two populations do not interact at all, then antibiotic use in one population cannot

64 affect resistance in the other. However, if two populations liberally exchange bacteria,

65 then the rates of antibiotic resistance in the two populations will be very similar,

66 regardless of whether their rates of antibiotic use differ greatly.

67

68 The effects of spillover also depend on population sizes. For example, two large 
69 populations will have most interactions within themselves, rather than between each

70 other. Spillover should therefore be most pronounced when considering small

71 populations and become less important for large populations. As mentioned above, a

72 single individual's risk of resistance is modulated by antibiotic use in their family or in

73 their healthcare facility. Spillover is also observed at the level of hospitals, as the level

74 of resistance in one hospital appears to be affected by resistance levels in nearby

75 hospitals as well as by antibiotic use rates in the surrounding communities (27-29).

76 Presumably, when examining ever larger populations, such as US Census tracts (30),

77 US states, or European countries, the effect of spillover will become less important.

78 However, the relationship between population size and spillover effects is not well

79 understood.

81 We hypothesized that US states and European countries, which are large populations

82 with relatively independent public health policies, may be subject to substantially lower

83 levels of antibiotic resistance spillover than family- or hospital-sized populations. This

84 hypothesis, if true, would mean that individual states or countries could act as

85 independent "laboratories" of antibiotic use and resistance. If not, it means that

86 outpatient antibiotic resistance policy must be national or international in order to

87 achieve its full effect. To evaluate this hypothesis, we first use mathematical models of

88 antibiotic use and resistance to make quantitative predictions about the effect of

89 spillover between populations as a function of their amount of mutual interaction. Then,

90 we search for signals of spillover in observational data of antibiotic use and resistance

91 in US states and European countries. 


\section{METHODS}

\section{Dynamical model of antibiotic resistance}

96 To examine how interactions between populations could theoretically affect the

97 association between antibiotic use and resistance, we used the within-host neutrality

98 (WHN) mathematical model presented by Davies et al. (31) and described in the

99 Supplemental Methods. Briefly, the model predicts the prevalence $\rho$ of antibiotic

100 resistance that results from an antibiotic use rate $t$ in a single, well-mixed population. To

101 verify that conclusions drawn from the WHN model are not specific to the model

102 structure, we also repeated all analyses with the "D-types" model of use and resistance

103 (32). We selected these two models because they demonstrate coexistence between

104 sensitive and resistant strains at equilibrium over a wide parameter space. Parameter

105 values and simulation methodology for both models are in the Supplemental Methods.

106 In the simulations, antibiotic use is measured as monthly treatments per capita and

107 resistance as the proportion of colonized hosts carrying resistant strains.

109 To conceptually frame and clarify the question of spillover, we simulated an antibiotic

110 stewardship intervention experiment using a structured host population approach

111 inspired by Blanquart et al. (33). We considered pairs of an intervention population with

112 antibiotic use rate $T_{\text {int }}$ and a control population with use rate $T_{\text {cont. }}$. To determine how

113 spillover affects the intervention's measured outcome, we modulated the proportion $\varepsilon$ of

114 each population's contacts that are in the other population. For $\varepsilon=0 \%$, the populations 
115 are completely separate. For $\varepsilon=50 \%$, contacts across populations are just as likely as

116 contacts within populations (Supplemental Methods). We varied $\varepsilon$ between $0 \%$ and

$11750 \%$, and we varied the difference in use $\Delta t=T_{\text {cont }}-T_{\text {int }}$ between 0 and 0.15 treatments

118 per person per month while keeping the average use $0.5 \times\left(T_{\text {cont }}+T_{\text {int }}\right)$ fixed at 0.125 ,

119 reflecting the range of antibiotic use rates in the original model presentations.

121 Observational data

122 We examined antibiotic use and resistance for 3 pathogen-antibiotic combinations: $S$.

123 pneumoniae and macrolides, S. pneumoniae and $\beta$-lactams, and Escherichia coli and

124 quinolones. We considered these 3 combinations because they are the subject of many

125 modeling $(31,32)$ and empirical studies $(5,15)$.

127 Observational data were drawn from 3 sources. First, we used MarketScan (34) and 128 ResistanceOpen (35) as previously described (7). The MarketScan data includes

129 outpatient pharmacy antibiotic prescription claims for 62 million unique people during

130 2011-2014. ResistanceOpen includes antibiotic resistance data collected during 2012-

1312015 from 230 hospitals, laboratories, and surveillance units in 44 states. Second, we

132 used the QuintilesIMS Xponent database (36) and the US Centers for Disease Control

133 and Prevention's (CDC) National Healthcare Safety Network (NHSN) (37). The Xponent

134 data includes state-level data on US quinolone use during 2011-2014. NHSN includes

135 state-level data on quinolone resistance among $E$. coli catheter-associated urinary tract

136 infections during 2011-2014. Third, we used the European Center for Disease

137 Prevention and Control's (ECDC) ESAC-Net antimicrobial consumption database (38) 
138 and EARS-Net Surveillance Atlas of Infectious Disease (39) for 2011-2015. The ESAC-

139 Net data includes country-level outpatient antibiotic use data provided by WHO and

140 Ministries of Health from member countries. The EARS-Net data includes country-level

141 resistance data. In the observational data, we quantified antibiotic use as yearly

142 treatments per capita and resistance as the proportion of collected isolates that were

143 non-susceptible. Further details about preparation of these data sources and their

144 availability are in the Supplemental Methods.

146 We excluded the S. pneumoniae resistance to $\beta$-lactams in US states from the analysis

147 because, in previous work using the same primary datasets, the point estimate for the

148 use-resistance relationship was negative (14).

150 Use-resistance relationships by populations' adjacency

151 To test the theoretical prediction that the same difference in antibiotic use will be

152 associated with smaller differences in antibiotic resistance when two populations (US

153 states or European countries) have stronger interactions, we tested whether the use-

154 resistance association is weaker in adjacent pairs of populations, which presumably

155 have more cross-population contacts, compared to non-adjacent populations. Two

156 populations were considered adjacent if they share a land or river border (40-42).

158 We quantified the use-resistance association as the percentage point difference (i.e.,

159 absolute risk difference) in resistance (proportion of non-susceptible isolates) divided by

160 the difference in antibiotic use. We summarized use-resistance associations among 
161 adjacent pairs and non-adjacent pairs of populations using the median value. Because

162 use-resistance associations between pairs of populations are correlated, we used the

163 jackknife method to compute confidence intervals on the difference in medians between

164 groups. We used the Mann-Whitney $U$ test to compute statistical significance. Because

165 our theoretical results suggested the ratio of percentage points difference in resistance

166 divided by difference in antibiotic use rates was a predictable function of the degree of

167 population mixing, we considered only this functional form for the use-resistance

168 association.

169

170 Use-resistance associations by interactions

171 Because adjacency might be too coarse measure of populations' interactions to detect

172 spillover, we performed a similar analysis as above, but predicting the use-resistance

173 association between populations using transportation data. For US states, we used

174 inter-county commuting statistics from the US Census (43). For European countries, we

175 used inter-country passenger flight data from Eurostat (44). Rather than trying to infer a

176 precise mathematical relationship between transportation statistics and epidemiological

177 contacts, we used a nonparametric approach: we assumed that pairs of populations

178 with relatively little inter-population transportation also have relatively few inter-

179 population contacts, but we infer only the rank ordering of inter-population contacts, not

180 their magnitudes. Specifically, we first converted the matrix of the number of counts

181 (workers in the commuting data and passengers in the flight data) from each population

182 to every other population into the proportion of counts moving from one population to

183 another (i.e., divided each row by its sum), then symmetrized the resulting matrix (taking 
184 the elementwise average of the matrix and its transpose), and finally converted the

185 resulting values into ranks. We assumed that intra-population interactions outnumber

186 inter-population interactions and so set diagonal entries, which represent within-

187 population interactions, to the highest rank. We measured the association between

188 ranked interactions and use-resistance associations using the nonparametric

189 Spearman's correlation, computed confidence intervals using the jackknife method, and

190 tested for statistical significance using the Mantel test with 999 permutations. To

191 quantify the effect of spillover, we compared the median use-resistance associations

192 among the top and bottom decile of ranked interactions.

194 Simulations and observational analyses were made using R (version 3.6.0) (45). The

195 Mantel test used the vegan package (46). Multiple hypotheses were accounted for using

196 Benjamini-Hochberg false discovery rate.

\section{RESULTS}

200 In simulations of two populations, representing an intervention and control group,

201 interactions between the two populations attenuated the effect of the intervention

202 (Figure 1). With increasing interaction strength, the same intervention, that is, the same

203 difference in antibiotic use between the populations, was associated with a smaller

204 difference in antibiotic resistance. The difference in resistance between populations

205 increases with the difference in antibiotic use (Figure 1d), but the use-resistance

206 association, measured as the ratio of the difference in resistance to the difference in 
207 use, depends strongly on the interaction strength (Figure 1e). Thus, spillover between

208 populations attenuates the measured use-resistance association.

210 The precise relationship between $\varepsilon$, the proportion of each population's contacts that are

211 in the other population, and the attenuation of the use-resistance association depended

212 on the choice of mathematical model (Supplemental Table 1, Supplemental Figure 1).

213 For $\varepsilon=1 \%$, the use-resistance declined by approximately $30 \%$ in the WHN model and

214 more than $60 \%$ in the "D-types" model. In other words, the models predict that as few as

$2151 \%$ of contacts need to be across populations, rather than within populations, to cause

216 the observed effect of an antibiotic stewardship intervention to shrink by one-third, or

217 even half.

219 To test whether spillover is important at the scale of US states or European countries,

220 we measured use-resistance associations between pairs of populations in 6

221 combinations of pathogen species, antibiotic class, and data source (Figure 2). We

222 reasoned that if spillover is relevant at these scales, then pairs of states or countries

223 with stronger interactions would have detectably weaker use-resistance associations.

225 We first tested whether pairs of physically adjacent populations (e.g., Massachusetts

226 and Connecticut) had weaker use-resistance associations than non-adjacent

227 populations (e.g., Massachusetts and Alaska). In 5 of 6 pathogen/antibiotic/dataset

228 combinations, the median use-resistance association was smaller among adjacent

229 populations than among non-adjacent populations (Figure 3), but in no case was the 
230 difference statistically significant after multiple hypothesis correction (Supplemental

231 Table 2). Point estimates of the relative difference in median use-resistance

232 associations between adjacent populations were $18 \%$ to $50 \%$ weaker than between

233 non-adjacent populations (excepting S. pneumoniae with $\beta$-lactams, which was an

234 outlier), consistent with the theoretical modeling results showing a $50 \%$ reduction in the

235 use-resistance association for populations with approximately $1 \%$ of interactions across

236 populations (Supplemental Table 1).

238 Next, to account for the possibility that adjacency was too coarse a measure for

239 interactions between populations, we instead used a rank-ordered estimate of

240 interactions using US commuting and European airline passenger flows (Supplemental

241 Figure 2). In 4 of 6 dataset/pathogen/antibiotic combinations, the nonparametric

242 association between increased inter-population interactions and decreased use-

243 resistance associations was statistically significant, thus confirming the general trend

244 observed in the adjacency analysis (Figure 4, Supplemental Table 3). The weakest

245 significant result was for $S$. pneumoniae and macrolides in the

246 MarketScan/ResistanceOpen dataset (Spearman's $\rho$ 0.07, 95\% jackknife confidence

247 interval -0.03 to $0.18 ; p=0.028$, Mantel test), and the strongest was for $E$. coli and

248 quinolones in the Xponent/NHSN dataset $(\rho=0.13,95 \%$ jackknife confidence interval

2490.007 to $0.25 ; p=0.001)$. The correlation for $S$. pneumoniae and macrolides in the

250 ECDC data has a point estimate suggesting spillover but was not statistically significant,

251 while the correlation for $S$. pneumoniae and $\beta$-lactams in the ECDC data had the

252 opposite point estimate, consistent with the adjacency analysis (Figure 3). 
254 Finally, to quantify the effect of increased interactions on the observed use-resistance

255 associations, we compared the use-resistance associations in pairs of populations

256 within the lowest decile of interactions against those in the highest decile, using the

257 same approach as for the adjacency analysis above (Supplemental Table 4). In 5 of 6

258 dataset/pathogen/antibiotic combinations, the point estimate for the different in use-

259 resistance associations was consistent with spillover, with a weaker association among

260 pairs of populations with greater interactions. In those 5 cases, the point estimates

261 ranged from a $18 \%$ reduction up to a $75 \%$ reduction in use-resistance associations

262 among the highest-interacting pairs of populations, compared to the lowest-interacting

263 populations.

\section{DISCUSSION}

267 We used theoretical models to show that interactions between two populations can

268 attenuate the observed use-resistance association. In simulations, the quantitative

269 relationship between inter-population interactions and the attenuation of the use-

270 resistance association was dependent on the theoretical model used. However, we

271 found that, in two models of the use-resistance association, having on the order of $1 \%$

272 of interactions between a control and intervention population was sufficient to attenuate

273 the observed effect of theoretical stewardship intervention by $50 \%$, relative to a situation

274 where the two populations were completely isolated. These theoretical results suggest

275 that even small numbers of interactions could lead to substantial spillover. 
277 When examining observational antibiotic use and resistance data from US states and

278 European countries, we did not detect a robust signal of spillover among pairs of

279 adjacent populations, as opposed to non-adjacent pairs, even across 3 pathogen-

280 antibiotic combinations in 3 separate datasets. However, when using more fine-grained

281 transportation data to estimate the relative ranking of epidemiological contacts between

282 those populations, we found a correlation between increased interactions and

283 attenuated use-resistance associations. Pairs of populations in the highest decile of

284 inter-population interactions, that is, those most subject to spillover, had use-resistance

285 associations on the order of $50 \%$ weaker than pairs in the lowest decile of interactions.

286 The 2 pathogen/antibiotic dataset combinations with data not indicative of spillover,

287 namely S. pneumoniae and $\beta$-lactams and macrolides in the ECDC data, may have not

288 shown the same signal as other cases because of the smaller number of populations in

289 those cases (27, versus 28 to 50 in the other cases) led to insufficient statistical power

290 or potentially because the biology or epidemiology of S. pneumoniae resistance in these

291 cases is somehow different and does not exhibit spillover.

293 These theoretical and empirical results suggest that spillover is relevant at the level of

294 US states and European countries. This finding has important ramifications. First,

295 attempts to attribute changes in a population's level of antibiotic resistance to changes

296 in that population's rates of antibiotic use may lead to inaccurate conclusions unless use

297 and resistance in surrounding populations is accounted for. Second, state- or country-

298 level antibiotic stewardship pilot studies may substantially underestimate the potential 
299 reduction in antibiotic resistance that would follow from a reduction in antibiotic use if

300 that reduction were implemented at a larger scale. Third, mass drug administration trials

301 may lead to elevated levels of antibiotic resistance in the control populations if those

302 populations are not entirely separated from the intervention population. Finally, spillover

303 can at least partly explain why use-resistance associations at the level of US states or

304 European countries are sometimes difficult to detect and, when they are detected, are

305 sometimes weaker than expected $(5,11,14)$. Furthermore, spillover means that

306 theoretical models of antibiotic use and resistance that treat US states or European

307 countries as epidemiologically independent populations will not accurately represent the

308 dynamics of resistance (33).

309

310 Our study has several limitations. First, we interpreted the theoretical results and

311 ecological data as if the association between antibiotic use and resistance were causal

312 and deterministic. However, decreases in the use of an antibiotic may not necessarily

313 lead to declines in resistance to that antibiotic in a target pathogen $(12,47-49)$. We do

314 not address co-resistance and cross-selection $(50,51)$, and we assumed that resistance

315 equilibrates on a timescale comparable to an intervention. Previous research has shown

316 that resistance among E. coli, S. pneumoniae, N. gonorrhoeae and other organisms can

317 respond to changes in antibiotic use on the timescale of months (52-55), but the

318 expected delay between a perturbation to antibiotic use and the resulting change in

319 resistance remains a subject of active study $(13,52,56,57)$. Nevertheless, the use of

320 ecological data was essential to addressing our hypothesis, as data from multiple

321 controlled, state- or country-wide experiments are not available. 
323 Second, our analyses attributed all differences in antibiotic resistance between

324 populations to differences in use across those populations and to interactions between

325 them. In fact, antibiotic resistance is associated with factors beyond antibiotic use

$326(6,58)$, and those factor are likely spatially correlated. In other words, closely interacting

327 populations might have more similar use-resistance associations because they tend to

328 be more similar with respect to other determinants of antibiotic use. Our estimates of the

329 correlation between inter-population interactions and the attenuation of use-resistance

330 relationships may therefore be overestimates. A more careful quantification of the

331 relative roles of spillover versus other spatially-correlated determinants of resistance is

332 required.

333

334 Third, our analysis only considered pairs of populations, when in fact spillover is

335 happening between all pairs of populations in our analysis simultaneously. We used the

336 pairs approach because it allowed for a simple theoretical model and a straightforward

337 comparison of theory with the observational data. However, more sophisticated

338 approaches that account for the network of spillover interactions will likely lead to more

339 refined characterizations of spillover.

340

341 Finally, analyses based on administrative entities like US states or European countries,

342 although logistically attractive "laboratories" of antibiotic stewardship, will always be

343 difficult to interpret because administrative entities average over important dimensions

344 of population structure like age (59), sexual networks (60), and race/ethnicity (61). Thus, 
345 use-resistance associations measured across states and countries may be different

346 from those that appear among geographically-proximate populations with dissimilar

347 antibiotic use rates, such as the sexes (62) and racial/ethnic groups (63).

349 We suggest 3 lines of investigation that could refine our understanding about the role of

350 spillover at levels of US states and European countries. First, further mathematical

351 modeling studies with more realistic structuring of the host population might articulate

352 more detailed theoretical expectations about the relationship between intervention scale

353 and spillover. For example, models could be parameterized with epidemiological

354 information about individuals' contacts and travel patterns, as has been done for other

355 infectious diseases (64). Second, meta-analysis of existing studies of use-resistance

356 relationships $(5,65,66)$, both experimental and observational, could potentially

357 determine the empirical relationship between intervention population size and the

358 importance of spillover. This kind of meta-analysis might reveal that populations other

359 than US states are feasible "laboratories" for resistance: it may be that cities, daycares,

360 schools, workplaces, or even families represent the optimal trade-off between

361 maximizing logistical feasibility and minimizing spillover. Finally, future experimental

362 outpatient antibiotic stewardship interventions should make careful and deliberate

363 decisions about the sizes and interconnectedness of the populations they target. We

364 hope that a better understanding of spillover will improve predictions about the future of

365 antibiotic resistance, the formulation of stewardship policy, the design of stewardship

366 interventions and antibiotic administration trials, and theoretical models of resistance. 


\section{References}

369 1. Review on Antimicrobial Resistance. Tackling drug-resistant infections globally:

370 final report and recommendations. 2016.

371 2. Public Health England. English Surveillance Programme for Antimicrobial

$372 \quad$ Utilisation and Resistance (ESPAUR). 2014.

373 3. Public Health Agency of Sweden, National Veterinary Institute. Consumption of $374 \quad$ antibiotics and occurrence of antibiotic resistance in Sweden.

375

376

377

378

379

380

381

382

383

384

385

386

387

388

389

390

391

392

393

394

395

396

397

398

399

400

401

4. US Centers for Disease Control and Prevention. Antibiotic Resistance Threats in the United Staes, 2013. 2013.

5. Bell BG, Schellevis F, Stobberingh E, Goossens H, Pringle M. A systematic review and meta-analysis of the effects of antibiotic consumption on antibiotic resistance. BMC Infect Dis. 2014 Jan 9;14:13.

6. MacFadden DR, McGough SF, Fisman D, Santillana M, Brownstein JS. Antibiotic resistance increases with local temperature. Nat Clim Change. 2018 Jun;8(6):5104.

7. Olesen SW, Barnett ML, MacFadden DR, Brownstein JS, Hernández-Diaz S, Lipsitch M, et al. The distribution of antibiotic use and its association with antibiotic resistance. eLife [Internet]. 2018 Dec 18 [cited 2018 Dec 19];7. Available from: https://elifesciences.org/articles/39435

8. Goossens H, Ferech M, Vander Stichele R, Elseviers M. Outpatient antibiotic use in Europe and association with resistance: a cross-national database study. The Lancet. 2005 Feb 12;365(9459):579-87.

9. van de Sande-Bruinsma N, Grundmann H, Verloo D, Tiemersma E, Monen J, Goossens $\mathrm{H}$, et al. Antimicrobial drug use and resistance in Europe. Emerg Infect Dis. 2008 Nov; 14(11):1722-30.

10. Livermore DM. The 2018 Garrod Lecture: Preparing for the Black Swans of resistance. J Antimicrob Chemother. 2018 Nov 1;73(11):2907-15.

11. Turnidge J, Christiansen $\mathrm{K}$. Antibiotic use and resistance--proving the obvious. Lancet Lond Engl. 2005 Feb;365(9459):548-9.

12. Arason VA, Gunnlaugsson A, Sigurdsson JA, Erlendsdottir H, Gudmundsson S, Kristinsson KG. Clonal Spread of Resistant Pneumococci Despite Diminished Antimicrobial Use. Microb Drug Resist. 2002 Sep;8(3):187-92.

13. Lipsitch M. The rise and fall of antimicrobial resistance. Trends Microbiol. 2001 Sep;9(9):438-44. 
402

403

404

405

406

407

408

409

410

411

412

413

414

415

416

417

418

419

420

421

422

423

424

425

426

427

428

429

430

431

432

433

434

435

436

14. Olesen SW, Barnett ML, MacFadden DR, Brownstein JS, Hernández-Díaz S, Lipsitch M, et al. The distribution of antibiotic use and its association with antibiotic resistance. eLife [Internet]. 2018 Dec 18 [cited 2019 Sep 23];7. Available from: https://elifesciences.org/articles/39435

15. Costelloe C, Metcalfe C, Lovering A, Mant D, Hay AD. Effect of antibiotic prescribing in primary care on antimicrobial resistance in individual patients: systematic review and meta-analysis. BMJ. 2010 May 18;340:c2096.

16. Harbarth S, Harris AD, Carmeli $Y$, Samore MH. Parallel analysis of individual and aggregated data on antibiotic exposure and resistance in gram-begative bacilli. Clin Infect Dis. 2001;33:1462-8.

17. Lipsitch M. Measuring and Interpreting Associations between Antibiotic Use and Penicillin Resistance in Streptococcus pneumoniae. Clin Infect Dis. 2001 Apr 1;32(7):1044-54.

18. Hannah EL, Angulo FJ, Johnson JR, Haddadin B, Williamson J, Samore MH. Drugresistant Escherichia coli, Rural Idaho. Emerg Infect Dis. 2005 Oct;11(10):1614-7.

19. Kalter HD, Gilman RH, Moulton LH, Cullotta AR, Cabrera L, Velapatiño B. Risk factors for antibiotic-resistant Escherichia coli carriage in young children in Peru: community-based cross-sectional prevalence study. Am J Trop Med Hyg. 2010 May;82(5):879-88.

20. Samore MH, Magill MK, Alder SC, Severina E, Morrison-De Boer L, Lyon JL, et al. High rates of multiple antibiotic resistance in Streptococcus pneumoniae from healthy children living in isolated rural communities: association with cephalosporin use and intrafamilial transmission. Pediatrics. 2001 Oct;108(4):856-65.

21. Gottesman B-S, Low M, Almog R, Chowers M. Quinolone Consumption by Mothers Increases Their Children's Risk of Acquiring Quinolone-Resistant Bacteriuria. Clin Infect Dis [Internet]. 2019 Aug 29 [cited 2019 Dec 2]; Available from: https://academic.oup.com/cid/advance-article/doi/10.1093/cid/ciz858/5556473

22. Lipsitch M, Samore MH. Antimicrobial use and antimicrobial resistance: a population perspective. Emerg Infect Dis. 2002;8:347-54.

23. Moellering RC. NDM-1 - A Cause for Worldwide Concern. N Engl J Med. 2010 Dec 16;363(25):2377-9.

24. Wang R, van Dorp L, Shaw LP, Bradley P, Wang Q, Wang X, et al. The global distribution and spread of the mobilized colistin resistance gene mcr-1. Nat Commun [Internet]. 2018 Dec [cited 2020 Jun 26];9(1). Available from: http://www.nature.com/articles/s41467-018-03205-z 
25. Bogoch II, Utzinger J, Lo NC, Andrews JR. Antibacterial mass drug administration for child mortality reduction: Opportunities, concerns, and possible next steps. Bhutta ZA, editor. PLoS Negl Trop Dis. 2019 May 23;13(5):e0007315.

26. Doan T, Arzika AM, Hinterwirth A, Maliki R, Zhong L, Cummings S, et al. Macrolide Resistance in MORDOR I - A Cluster-Randomized Trial in Niger. N Engl J Med. 2019 Jun 6;380(23):2271-3.

27. Cooper BS, Medley GF, Stone SP, Kibbler CC, Cookson BD, Roberts JA, et al. Methicillin-resistant Staphylococcus aureus in hospitals and the community: Stealth dynamics and control catastrophes. Proc Natl Acad Sci. 2004 Jul 6;101(27):10223-

28. MacFadden DR, Fishman DN, Hanage WP, Lipsitch M. The Relative Impact of Community and Hospital Antibiotic Use on the Selection of Extended-Spectrum Beta-lactamase-Producing Escherichia coli. Clin Infect Dis [Internet]. 2018 [cited 2018 Nov 29]; Available from: https://www.ncbi.nlm.nih.gov/pubmed/30462185

29. Knight GM, Costelloe C, Deeny SR, Moore LSP, Hopkins S, Johnson AP, et al. Quantifying where human acquisition of antibiotic resistance occurs: a mathematical modelling study. BMC Med. 2018 23;16(1):137.

30. Klevens RM, Caten E, Olesen SW, DeMaria A, Troppy S, Grad YH. Outpatient Antibiotic Prescribing in Massachusetts, 2011-2015. Open Forum Infect Dis [Internet]. 2019 May 1 [cited 2019 Sep 15];6(5). Available from: https://academic.oup.com/ofid/article/doi/10.1093/ofid/ofz169/5430941

31. Davies NG, Flasche S, Jit M, Atkins KE. Within-host dynamics shape antibiotic resistance in commensal bacteria. Nat Ecol Evol. 2019 Mar;3(3):440-9.

32. Lehtinen S, Blanquart F, Croucher NJ, Turner P, Lipsitch M, Fraser C. Evolution of antibiotic resistance is linked to any genetic mechanism affecting bacterial duration of carriage. Proc Natl Acad Sci. 2017 Jan 31;114(5):1075-80.

33. Blanquart F, Lehtinen S, Lipsitch M, Fraser $\mathrm{C}$. The evolution of antibiotic resistance in a structured host population. J R Soc Interface. 2018 Jun;15(143).

34. Truven Health MarketScan Database. Commercial Claims and Encounters. Ann Arbor, Ml; 2015.

35. MacFadden DR, Fisman D, Andre J, Ara Y, Majumder MS, Bogoch II, et al. A Platform for Monitoring Regional Antimicrobial Resistance, Using Online Data Sources: ResistanceOpen. J Infect Dis. 2016 Dec 1;214(suppl_4):S393-8.

36. US Centers for Disease Control and Prevention. Patient Safety Atlas - Outpatient Antibiotic Use [Internet]. [cited 2018 Oct 25]. Available from: https://gis.cdc.gov/grasp/PSA/indexAU.html 
37. US Centers for Disease Control and Prevention. Patient Safety Atlas - Antibiotic Resistance [Internet]. [cited 2018 Oct 25]. Available from: https://gis.cdc.gov/grasp/PSA/AboutTheData.html

38. European Centre for Disease Prevention and Control. Antimicrobial consumption database [Internet]. European Centre for Disease Prevention and Control. [cited 2018 Oct 25]. Available from: http://ecdc.europa.eu/en/antimicrobialconsumption/surveillance-and-disease-data/database

39. European Centre for Disease Prevention and Control. Surveillance Atlas of Infectious Diseases [Internet]. [cited 2018 Oct 25]. Available from: https://atlas.ecdc.europa.eu/public/index.aspx

40. US Census. County Adjacency File [Internet]. Available from: https://www.census.gov/geo/reference/county-adjacency.html

41. Correlates of War Project. Direct Contiguity Data, 1816-2016 (v3.2) [Internet]. Available from: http://correlatesofwar.org/data-sets/direct-contiguity

42. Stinnett DM, Tir J, Diehl PF, Schafer P, Gochman C. The Correlates of War (Cow) Project Direct Contiguity Data, Version 3.0. Confl Manag Peace Sci. 2002 Sep;19(2):59-67.

43. US Census. 2011-2015 5-Year ACS Commuting Flows [Internet]. Available from: https://www.census.gov/data/tables/2015/demo/metro-micro/commuting-flows2015.html

44. Eurostat. Air transport measurement [Internet]. Available from: https://ec.europa.eu/eurostat/cache/metadata/en/avia_pa_esms.htm

45. R Core Team. R: A language and environment for statistical computing [Internet]. Vienna; 2018. Available from: www.R-project.org

46. Oksanen J, Blanchet FG, Friendly M, Kindt R, Legendre $P$, McGlinn D, et al. vegan: Community Ecology Package. $R$ package version 2.5-6.

47. Hennessy TW, Petersen KM, Bruden D, Parkinson AJ, Hurlburt D, Getty M, et al. Changes in Antibiotic-Prescribing Practices and Carriage of Penicillin-Resistant Streptococcus pneumoniae: A Controlled Intervention Trial in Rural Alaska. Clin Infect Dis. 2002 Jun 15;34(12):1543-50.

48. Sundqvist M, Geli P, Andersson DI, Sjölund-Karlsson M, Runehagen A, Cars H, et al. Little evidence for reversibility of trimethoprim resistance after a drastic reduction in trimethoprim use. J Antimicrob Chemother. 2010 Feb;65(2):350-60.

49. Enne VI, Livermore DM, Stephens P, Hall LM. Persistence of sulphonamide resistance in Escherichia coli in the UK despite national prescribing restriction. Lancet Lond Engl. 2001 Apr 28;357(9265):1325-8. 
50. Pouwels KB, Freeman R, Muller-Pebody B, Rooney G, Henderson KL, Robotham $\mathrm{JV}$, et al. Association between use of different antibiotics and trimethoprimresistance: going beyond the obvious crude association. J Antimicrob Chemoth. 2018;

51. Tedijanto C, Olesen SW, Grad YH, Lipsitch M. Estimating the proportion of bystander selection for antibiotic resistance among potentially pathogenic bacterial flora. Proc Natl Acad Sci. 2018 Dec 18;115(51):E11988-95.

52. Olesen SW, Torrone EA, Papp JR, Kirkcaldy RD, Lipsitch M, Grad YH. Azithromycin Susceptibility Among Neisseria gonorrhoeae Isolates and Seasonal Macrolide Use. J Infect Dis [Internet]. 2018 [cited 2018 Nov 6]; Available from: http://academic.oup.com/jid/advance-article/doi/10.1093/infdis/jiy551/5098400

53. Dagan R, Barkai G, Givon-Lavi N, Sharf AZ, Vardy D, Cohen T, et al. Seasonality of Antibiotic-Resistant Streptococcus pneumoniae That Causes Acute Otitis Media: A Clue for an Antibiotic-Restriction Policy? J Infect Dis. 2008 Apr 15;197(8):1094102.

54. Sun L, Klein EY, Laxminarayan R. Seasonality and Temporal Correlation between Community Antibiotic Use and Resistance in the United States. Clin Infect Dis. 2012 Sep 1;55(5):687-94.

55. Blanquart $F$, Lehtinen $S$, Fraser $C$. An evolutionary model to predict the frequency of antibiotic resistance under seasonal antibiotic use, and an application to Streptococcus pneumoniae. Proc R Soc B. 2017 May 31;284(1855):20170679.

56. Dingle KE, Didelot X, Quan TP, Eyre DW, Stoesser N, Golubchik T, et al. Effects of control interventions on Clostridium difficile infection in England: an observational study. Lancet Infect Dis. 2017 Apr;17(4):411-21.

57. McCormick AW, Whitney CG, Farley MM, Lynfield R, Harrison LH, Bennett NM, et al. Geographic diversity and temporal trends of antimicrobial resistance in Streptococcus pneumoniae in the United States. Nat Med. 2003 Apr;9(4):424-30.

58. Collignon P, Beggs JJ, Walsh TR, Gandra S, Laxminarayan R. Anthropological and socioeconomic factors contributing to global antimicrobial resistance: a univariate and multivariable analysis. Lancet Planet Health. 2018 Sep;2(9):e398-405.

59. Mossong J, Hens N, Jit M, Beutels P, Auranen K, Mikolajczyk R, et al. Social contacts and mixing patterns relevant to the spread of infectious diseases. PLoS Med. 2008 Mar 25;5(3):e74.

60. Garett G, Hughes J, Anderson R, Stoner B, Aral S, Whittington W, et al. Sexual mixing patterns of patients attending sexually transmitted diseases clinics. Sex Transm Dis. 1996;23(3):248-57. 
545 61. Newman MEJ. Mixing patterns in networks. Phys Rev E Stat Nonlin Soft Matter Phys. 2003 Feb;67(2 Pt 2):026126.

547 62. Hicks LA, Bartoces MG, Roberts RM, Suda KJ, Hunkler RJ, Taylor TH, et al. US Outpatient Antibiotic Prescribing Variation According to Geography, Patient Population, and Provider Specialty in 2011. Clin Infect Dis. 2015 May 1;60(9):1308-16.

63. Olesen SW, Grad YH. Racial/Ethnic Disparities in Antimicrobial Drug Use, United States, 2014-2015. Emerg Infect Dis [Internet]. 2018 [cited 2018 Nov 6];24(11). Available from: https://wwwnc.cdc.gov/eid/article/24/11/18-0762_article

64. Charu V, Zeger S, Gog J, Bjørnstad ON, Kissler S, Simonsen L, et al. Human mobility and the spatial transmission of influenza in the United States. PLoS Comput Biol. 2017;13(2):e1005382.

65. Schechner V, Temkin E, Harbarth S, Carmeli Y, Schwaber MJ. Epidemiological interpretation of studies examining the effect of antibiotic usage on resistance. Clin

66. O'Brien KS, Emerson P, Hooper P, Reingold AL, Dennis EG, Keenan JD, et al. Antimicrobial resistance following mass azithromycin distribution for trachoma: a systematic review. Lancet Infect Dis [Internet]. 2018 Oct 3 [cited 2018 Nov 7]; Available from: 


\section{Disclaimers}

568 The views and opinions of the authors expressed herein do not necessarily state or

569 reflect those of the ECDC. The accuracy of the authors' statistical analysis and the

570 findings they report are not the responsibility of ECDC. ECDC is not responsible for

571 conclusions or opinions drawn from the data provided. ECDC is not responsible for the

572 correctness of the data and for data management, data merging and data collation after

573 provision of the data. ECDC shall not be held liable for improper or incorrect use of the

574 data.

575

576 Funding

577 This work was supported by the National Institutes of Health (grant number

578 U54GM088558 to ML). The funders had no role in study design, data collection and

579 interpretation, or the decision to submit the work for publication.

580

581 Acknowledgements

582 We thank Dr. Stephen M. Kissler for helpful comments on the manuscript.

583

584 
585 Figure 1. Interactions between populations attenuate the effect of interventions.

586 (a) Schematic of the 2-population model. (b) Results of simulations of the 2-population

587 WHN model for a modest intervention (difference in antibiotic use between populations

$588 \Delta T=0.05$ monthly treatments per capita; average of control and intervention treatment

589 rates 0.125$)$. As interaction strength ( $\varepsilon$, horizontal axis) increases, the difference in

590 antibiotic resistance between the two populations decreases. Dotted line shows

591 resistance level in populations before the intervention. (c) The same pattern holds for a

592 stronger intervention ( $\Delta T=0.1$, same average treatment rate). (d) In general, the

593 difference in resistance between populations ( $\Delta \rho$, vertical axis) increases with the

594 difference in antibiotic use ( $\Delta T$, horizontal axis). (e) However, in the WHN model, the

595 use-resistance relationship ( $\Delta \rho / \Delta T$, vertical axis) depends mostly on the interaction

596 strength $\varepsilon$ and is mostly independent of the difference in antibiotic use $\Delta T$.
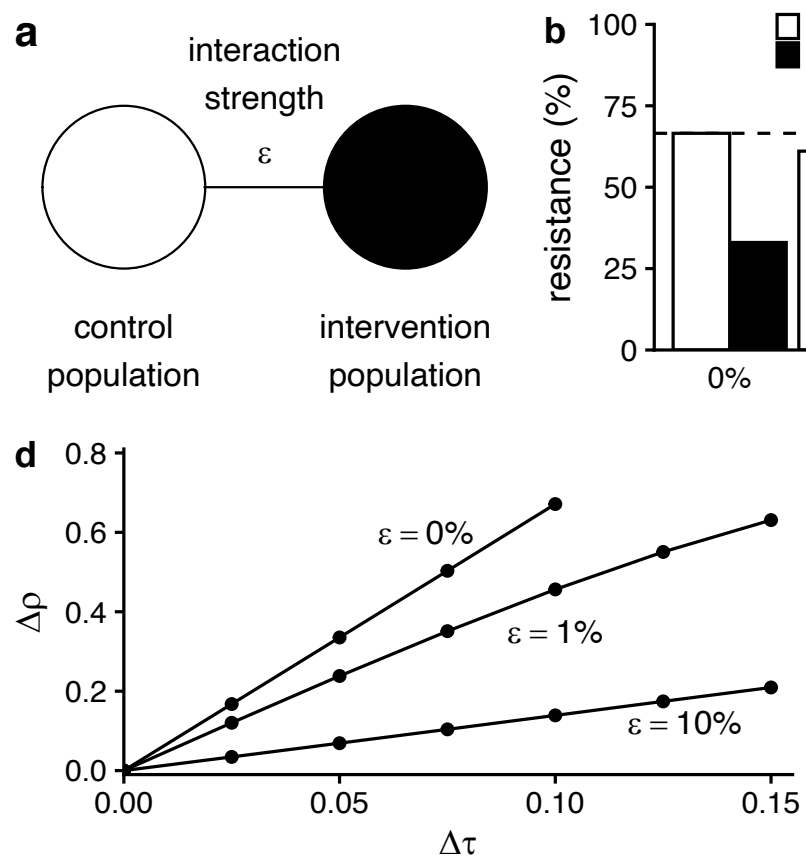
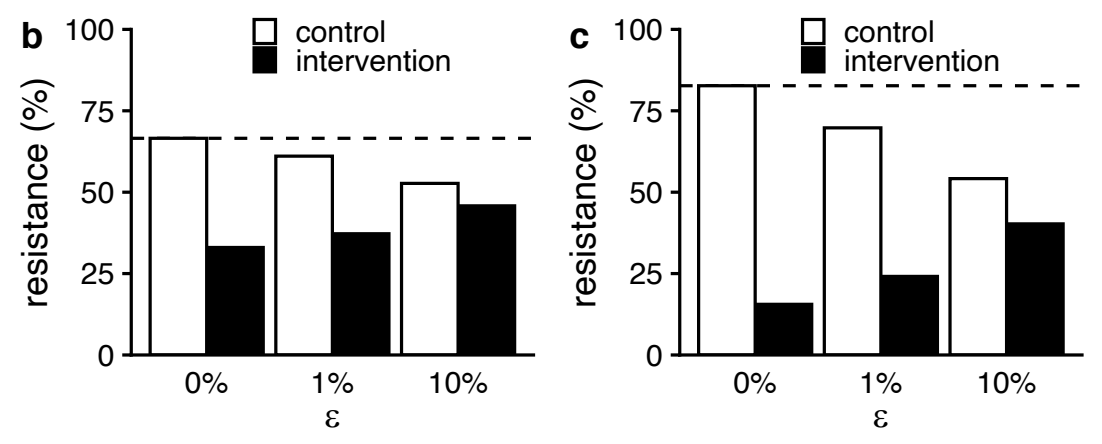

e

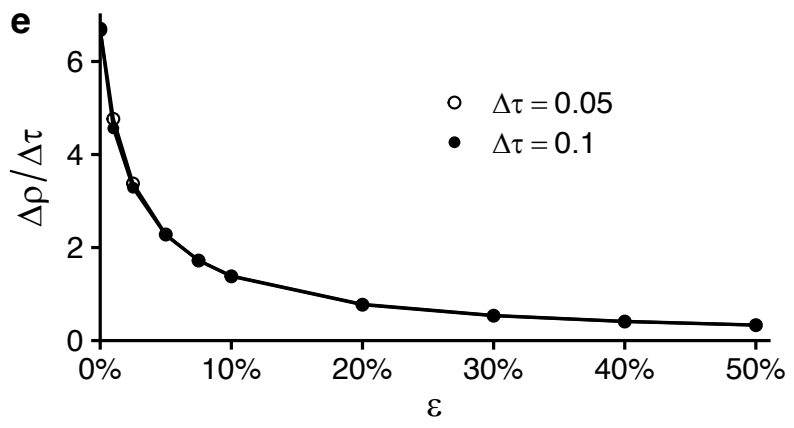


599 Figure 2. Use-resistance relationships across US states and European countries.

600 Each point represents antibiotic use and resistance in a US state (top row) or European

601 country (bottom row). Lines show simple linear regression best fit. Gray areas show

602 95\% confidence interval. Ec/q: E. coli and quinolones. Sp/m: S. pneunomiae and

603 macrolides. Sp/bl: S. pneumoniae and $\beta$-lactams. RO: ResistanceOpen. ECDC:

604 European CDC.
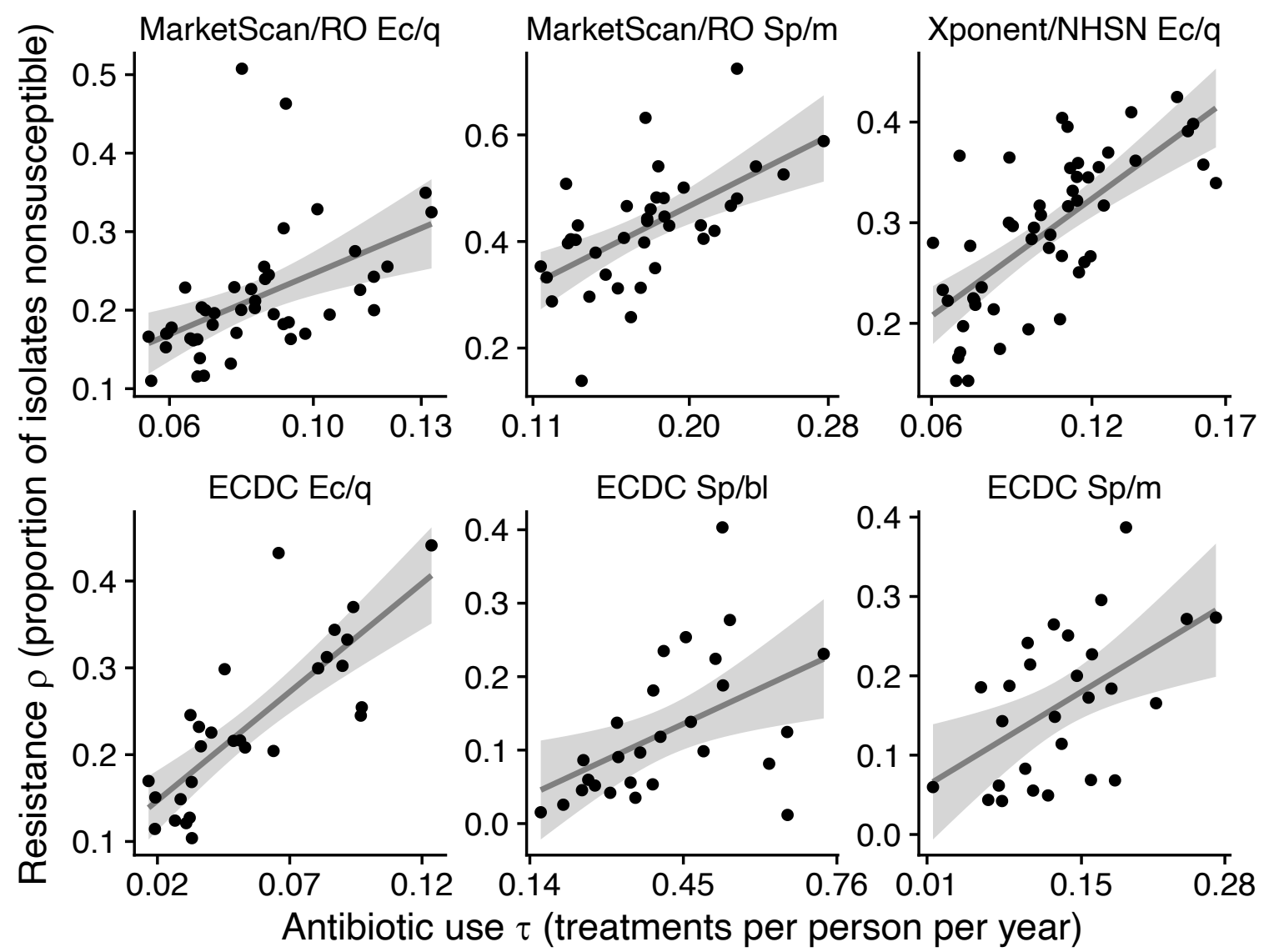

605 Antibiotic use $\tau$ (treatments per person per year) 
607 Figure 3. Use-resistance relationships by adjacency. Each point represents the use-

608 resistance association in populations (top row, US states; bottom row, European

609 countries), the same as those shown in Figure 2, arranged by whether the pair of

610 populations is physically adjacent. Physically adjacent populations tend to have weaker

611 use-resistance associations, but differences were not statistically significant. For visual

612 clarity, the vertical axes are truncated to show only the central $90 \%$ of data points. Ec/q:

613 E. coli and quinolones. Sp/m: S. pneunomiae and macrolides. Sp/bl: S. pneumoniae

614 and $\beta$-lactams. RO: ResistanceOpen. ECDC: European CDC.

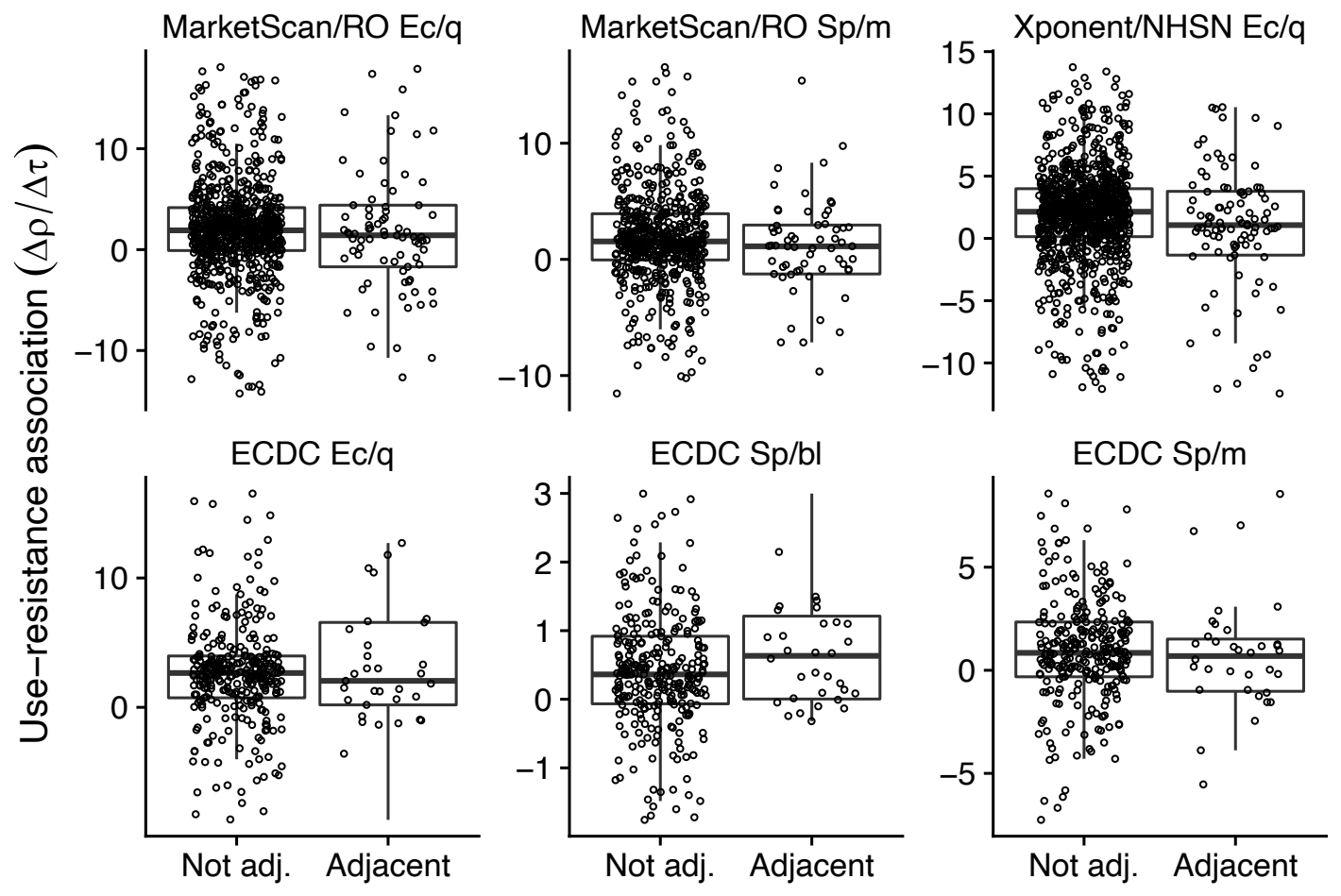

615 
617 Figure 4. Use-resistance associations by ranked interaction. Each point represents

618 the use-resistance association in a pair of US states (top row) or European countries

619 (bottom row), the same pairs as shown in Figure 3, rank ordered by increasing inter-

620 population interaction as inferred from transportation data. For visual clarity, the

621 horizontal axes are truncated to exclude outliers. The dashed red line is a visual

622 illustration of how increasing interaction is correlated with decreasing use-resistance

623 associations (robust regression; compare Supplemental Tables 3 and 4). The asterisk

$624\left(^{*}\right)$ indicates a statistically significant association between increased interaction and

625 decreased use-resistance relationship (Supplemental Table 3). Ec/q: E. coli and

626 quinolones. Sp/m: S. pneunomiae and macrolides. Sp/bl: S. pneumoniae and $\beta-$

627 lactams. RO: ResistanceOpen. ECDC: European CDC.

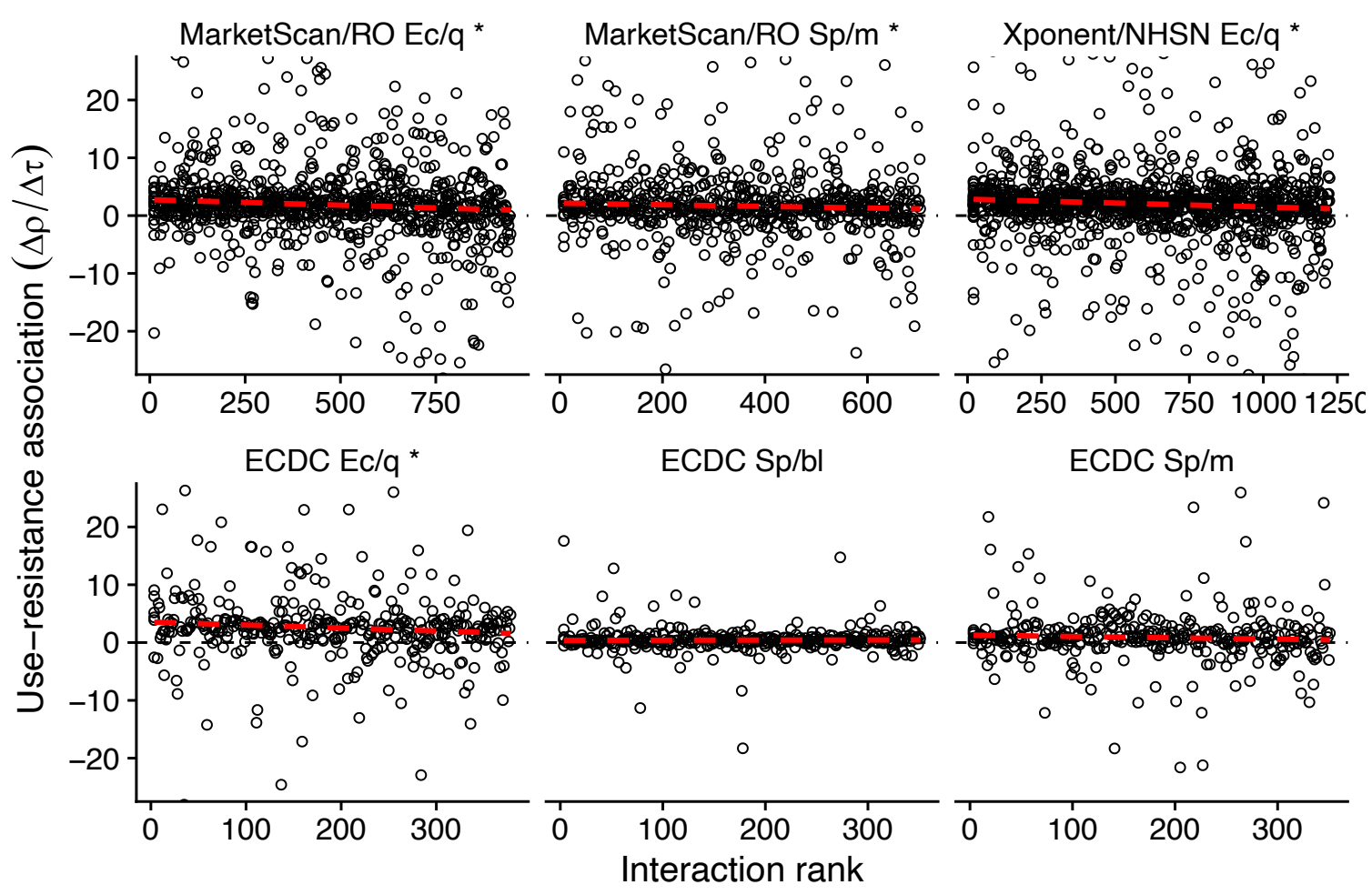

628

629 